\title{
Generation of Temporal Low Noise Laser Pulses for Investigating of Laser Peening Process
}

\author{
Masayuki SUZUKI, ${ }^{1}$ Hiromitsu KIRIYAMA,${ }^{2}$ Izuru DAITO,${ }^{2}$ Hajime OKADA,, 2 Yoshihiro OCHI, ${ }^{2}$ \\ Masatoshi SATO, ${ }^{3}$ Yoshinori TAMAOKI, ${ }^{4}$ Takehiro YOSHII, Junya MAEDA, ${ }^{3}$ Shinichi MATSUOKA, ${ }^{3}$ \\ Hirofumi KAN, ${ }^{4}$ Paul R. BOLTON, ${ }^{2}$ Akira SUGIYAMA, ${ }^{2}$ and Kiminori KONDO ${ }^{2}$ \\ ${ }^{1}$ Ophthalmology and Advanced Laser Medical Center Faculty of Medicine, Saitama Medical University \\ 38 Morohongo Moroyama-machi, Iruma-gun, Saitama 350-0495 \\ ${ }^{2}$ Quantum Beam Science Directorate and Kansai Photon Science Institute, Japan Atomic Energy Agency, \\ 8-1-7 Umemidai, Kizugawa-city, Kyoto 619-0215 \\ ${ }^{3}$ Development Bureau, Hamamatsu Photonics K.K., 5000 Hirakuchi, Hamakitaku, Hamamatsu-city, Shizuoka 434-8601 \\ ${ }^{4}$ Industries Development Laboratory Power Laser Development Hamamatsu Photonics K.K., \\ 1820, Kurematsu-cho, Nishi-ku, Hamamatsu City, Shizuoka Pref., 431-1202
}

(Received February 7, 2014)

\begin{abstract}
We have demonstrated an optical parametric chirped pulse amplification (OPCPA)/Yb:YAG ceramic thin disk hybrid laser system having the hundred $\mathrm{mJ}$ pulse energy level with sub-picosecond duration and high temporal contrast. At an input chirped-pulse energy of $3.8 \mathrm{~mJ}$ from an OPCPA preamplifer an output energy of $130 \mathrm{~mJ}$ has been generated from a multipass laser-diode (LD) pumped $\mathrm{Yb}$ :YAG ceramic thin disk amplifier. A recompressed pulse duration of 450 fs with a contrast level of less than $7.2 \times 10^{-9}$ at $150 \mathrm{ps}$ before the main pulse has been obtained. The contrast level was the highest value achieved in a Yb:YAG chirped pulse amplification (CPA) laser system at the hundred $\mathrm{mJ}$ level. This laser system is well-suited to studying laser matter interactions in the laser peening mechanism.
\end{abstract}

Key Words: High temporal contrast laser system, Optical parametric chirped pulse amplifier, Yb:YAG thin disk, Laser matter interaction, Laser peening

\section{Introduction}

Residual stress compression on a metal surface by laser peening is widely applied to large aircraft components and power generation parts. ${ }^{1,2)}$ To date, laser peening has been mainly demonstrated with pulses of nanosecond duration. A new approach using femtosecond laser pulses is being investigated because larger shockwaves can be generated at higher laser peak intensity. ${ }^{3)}$ Characterization of shockwave generation with femtosecond laser pulses is needed to understand the femtosecond laser peening mechanism.

A femtosecond laser system generally consists of an oscillator, a pulse stretcher, a regenerative amplifier, a multipass amplifier, and a pulse compressor. Temporal noise in the laser pulse is due to prepulses and amplified spontaneous emission (ASE) levels that can be significant in a regenerative amplifier. When the ASE or prepulse is focused on a target material before the femtosecond laser pulse, a preplasma is generated on target surface ${ }^{4)}$ which can make it difficult to distinctively characterize the high peak intensity femtosecond laser plasma interaction. Consequently, laser pulses with low temporal noise (i.e. high temporal contrast), achieved by the reduction of ASE and prepulses levels, are preferable for understanding the laser peening mechanism.

Optical parametric chirped pulse amplification (OPCPA) is one of the most efficient techniques for reducing ASE and prepulse levels in a preamplifier. ${ }^{5)}$ Actually, these levels can be reduced by at least two orders of magnitudes compared with a typical regenerative amplifier. ${ }^{6}$ Therefore the OPCPA preamplifier have been developed to generate high quality seed pulses in the OPCPA/Ti:sapphire (or glass) hybrid laser systems for high peak intensity laser (in the petawatt class). ${ }^{7-11)}$ In laser matter interaction experiments preplasma reduction has been demonstrated. ${ }^{12}$

As a gain medium for generation of femtosecond laser pulses, Ti:sapphire crystals have been mainly used. However this system is large and of low efficiency. Yb:YAG CPA is eminently suitable for a compact high peak power, high repetition rate laser system because of its high efficiency that is attributed to direct laser diode pumping and a correspondingly small system thermal load. ${ }^{13)}$ Furthermore recent progress of large aperture transparent polycrystalline ceramic with high doping concentrations enable high intensity laser pulse operation at high repetition-rates. ${ }^{14,15)}$ Currently, Yb based CPA systems have been demonstrated where typically a regenerative amplifier has been used. ${ }^{16-19)}$ ASE and prepulse levels can be significant with this preamplifier.

Here we present a demonstration of an OPCPA/Yb:YAG ceramic thin disk hybrid laser system with an output energy having the hundred $\mathrm{mJ}$ level, sub-picosecond pulse duration and high temporal contrast.

\section{Experiments}

Figure 1 shows the experimental diagram for our OPCPA/ $\mathrm{Yb}: Y A G$ hybrid laser system. The laser system consists of an 


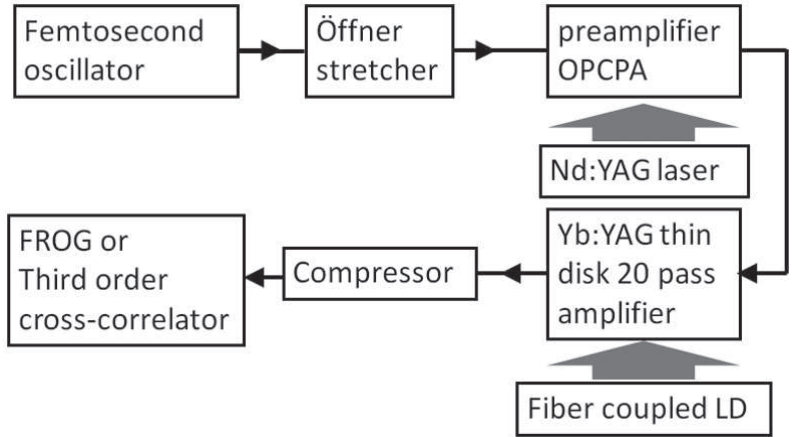

Fig. 1 Configuration of the OPCPA/Yb:YAG hybrid laser system.

oscillator, an aberration free (Öffner) stretcher, an OPCPA preamplifier, a multi-pass Yb:YAG ceramic thin disk amplifier, and a compressor.

\subsection{Frontend and OPCPA pre-amplifier}

From the oscillator (ORIGAMI-HP, Onefive) near transform-limited pulses of energy of $25 \mathrm{~nJ}$ are delivered at a $40 \mathrm{MHz}$ repetition-rate (i.e. $1 \mathrm{~W}$ average power). The central wavelength of this laser pulse is $1029 \mathrm{~nm}$ with the spectral bandwidth of $5 \mathrm{~nm}$ at FWHM. The pulse duration of $200 \mathrm{fs}$ is measured by a commercial FROG (GRENOUILLE UPM-10-100, Swamp Optics). The Öffner stretcher (consisting of a gold coated grating by HORIBA Jobin Yvon SAS, a spherical mirror and a concave mirror) extends the seed pulse duration to $1 \mathrm{~ns}$ with $13.8 \mathrm{~nJ}$ energy (stretcher energy efficiency of 55\%). Following the stretcher a commercial pulse selector (UPS 068, LEYSOP) extracts $10 \mathrm{~Hz}$ seed pulses that are synchronized to a $10 \mathrm{~Hz} \mathrm{Nd:YAG} \mathrm{pump} \mathrm{laser} \mathrm{(Quanta-Ray}$ PRO 250, Spectra Physics). Second harmonic generation (SHG) at $532 \mathrm{~nm}$ of a Nd:YAG laser is used to pump the OPCPA. Synchronized pump and seed pulses enter the OPCPA unit which is a three-stage configuration using anti-reflection coated BBO (cut at an angle of $23.8^{\circ}$ ) at each stage. The transverse dimensions of all three $\mathrm{BBO}$ crystals are $7 \mathrm{~mm}$ $\times 7 \mathrm{~mm}$.

The thicknesses of the first two and the third crystals are 20 $\mathrm{mm}$ and $16 \mathrm{~mm}$, respectively. The pump laser can deliver a maximum SHG pulse energy of $450 \mathrm{~mJ}$ within $8 \mathrm{~ns}$ (FWHM) at $10 \mathrm{~Hz}$. The beam sizes at the BBO surface for both the pump and the seed beams are $3.7 \mathrm{~mm}\left(1 / e^{2}\right)$. For all three BBO stages, the seed and pump beam lines are non-collinear

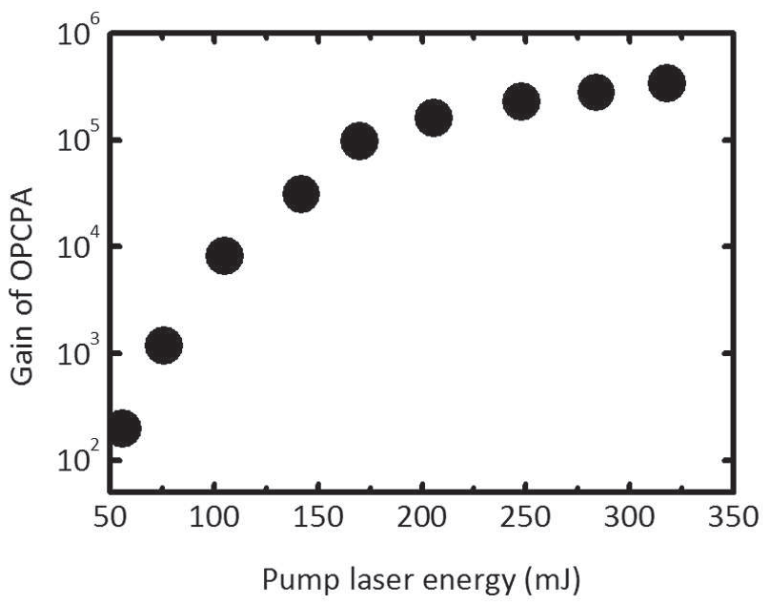

Fig. 2 Single pass gain of OPCPA preamplifier as a function of the pumping laser pulse energy.

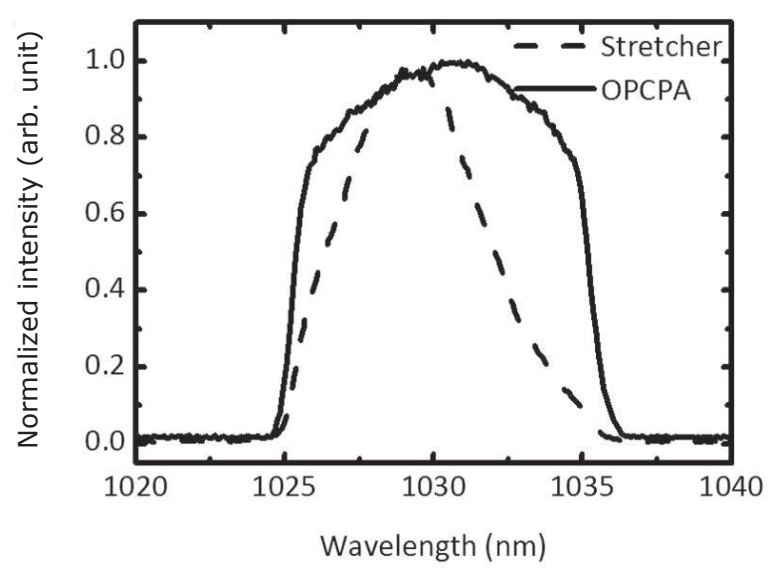

Fig. 3 OPCPA (solid black curve) and stretcher (solid gray curve) exit spectra.

and separated by an angle of $1.92^{\circ}$. The mismatching between pump and seed pulses would generate the parametric fluorescence. To suppress the parametric fluorescence as much as possible, we made the following amendments in the OPCPA preamplifier: (1) lowering the parametric gain while maintaining sufficient output energy, (2) maintaining a precise spatial overlap between the pump and stretched seed beams, (3) using wedge output surface for each nonlinear crystal. Figure 2 illustrates the dependence of OPCPA gain on pump energy. These results were obtained using a large-area photoreceiver with an oscilloscope (Wave Runner $104 \mathrm{Xi}$, LeCroy). The single pass OPCPA gain exceeds $10^{5}$ such that the output energy is amplified to $3.8 \mathrm{~mJ}$ with only a $13.8 \mathrm{~nJ}$ input. ${ }^{20)}$

Figure 3 shows the exit spectra from the OPCPA and stretcher. The bandwidth of this amplified laser pulse is 10.7 $\mathrm{nm}$ (FWHM). Spectral broadening and associated pulse shaping are optimized in the OPCPA unit by the phase matching established with the BBO tuning angle as reported ${ }^{5)}$ in detail. Consequently, unlike the peaked symmetric spectral profile of the incident seed, the spectral profile of the amplified laser pulse is that of a symmetric flat top.

\subsection{Main amplifier}

After preamplification by the OPCPA, the laser pulse was further amplified in multiple passes through the $\mathrm{Yb}$ :YAG (doping level at 7 at\%) ceramic thin disk (Konoshima Chemical). The ceramic thin disk of $0.6 \mathrm{~mm}$ thickness is in optical

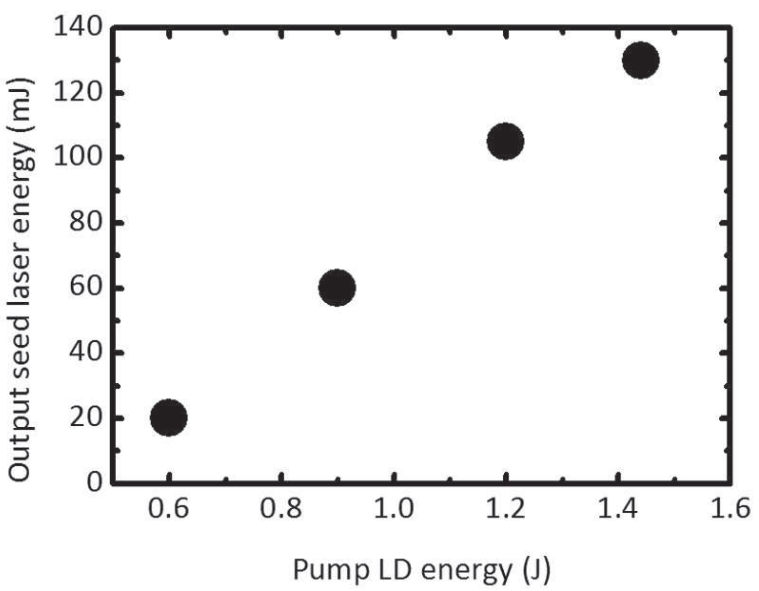

Fig. 4 The output energy of Yb:YAG ceramic thin disk 20-pass amplifier as a function of the pump energy of LD at $940 \mathrm{~nm}$. 
contact with a non-doped YAG disk of $1 \mathrm{~mm}$ thickness and 10 $\mathrm{mm}$ diameter. This ceramic disk was pumped by a quasi-CW (Q-CW) fiber coupled laser diode (LD) (LA1216, Hamamatsu Photonics) with a pulse energy of $2 \mathrm{~J}$ within a $2 \mathrm{~ms}$ duration ( $\mathrm{kW}$ peak power). The details of the pumping system using the LD (TDM 1.0 HP, Dausinger+Giesen) for the Yb:YAG thin disk has been described elsewhere. ${ }^{21)}$ Absorption of the LD pump light was calculated to be $95 \%$ by numerical calculation. The pump fluence on the ceramic is $1.9 \mathrm{~J} / \mathrm{cm}^{2}$. The diameters of the LD pump spot and seed laser pulse are $4.5 \mathrm{~mm}$ and $3.8 \mathrm{~mm}$, respectively. The single pass loss is $9 \%$ without LD pumping. The first 10 -pass the amplified seed pulse was reflected by using a concave mirror with a radius of curvature of $20 \mathrm{~m}$ to collimate a beam divergence. After 20-pass the amplified pulse was extracted with a thin film polarizer (TFP). Figure 4 shows the output energy after 20-pass through the Yb:YAG ceramic disk as a function of the LD pump energy. The amplified laser pulse energy increases with LD pump energy. With an input laser energy of $3.8 \mathrm{~mJ}$ the maximum output energy from the thin disk amplifier after 20-pass is $130 \mathrm{~mJ}$ at a $10 \mathrm{~Hz}$ repetition-rate with good beam quality. The optical efficiency from LD energy to amplified laser pulse energy is $9.6 \%$. Figure 5 shows the amplified transverse beam profile diameter of $4.5 \mathrm{~mm}\left(1 / e^{2}\right)$.

The spectral bandwidth of amplified pulse is $2.5 \mathrm{~nm}$ before pulse compression as shown in Fig. 6.

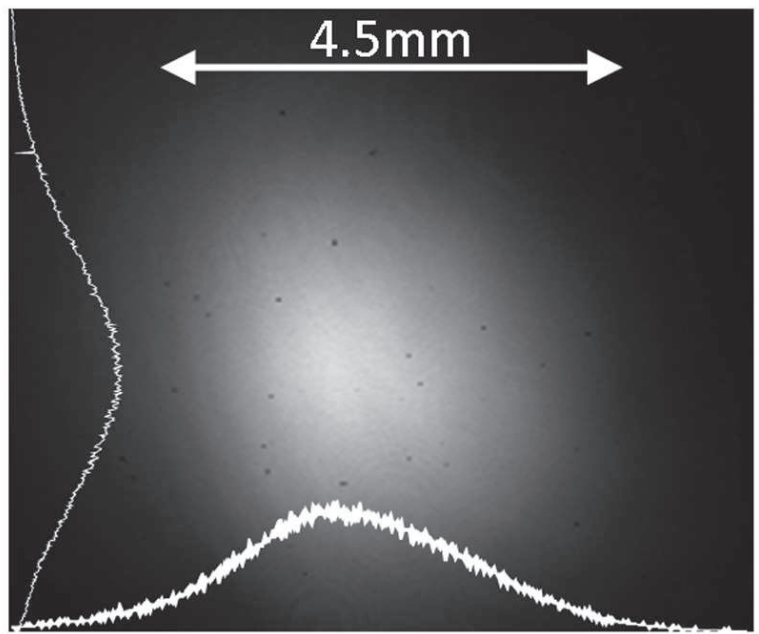

Fig. 5 Amplified laser beam profile of Yb:YAG ceramic thin disk 20-pass amplifier.

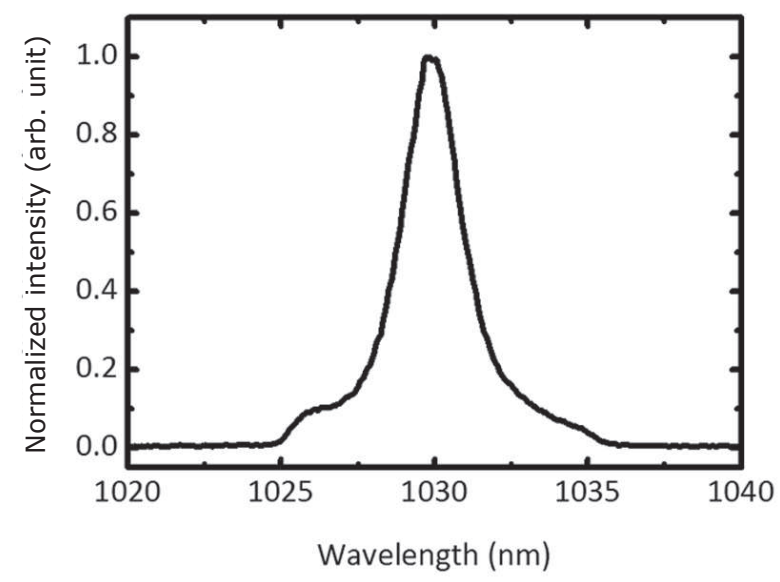

Fig. 6 Spectrum of amplified laser. The bandwidth of spectral is $2.5 \mathrm{~nm}$ (FWHM).

\subsection{Compressor}

The output laser pulse from Yb:YAG ceramic thin disk amplifier is subsequently recompressed by a gold coated grating pair of 1740 grooves/mm (HORIBA Jobin Yvon SAS). The pulse compressor consisted of two parallel gold coated diffraction gratings, which had a measured diffraction efficiency of $\sim 93 \%$. The whole efficiency of the pulse compressor was measured to be $\sim 73 \%$. As can be seen in Fig. 7, the compressed pulse duration was measured to be 450 fs by using FROG. Assuming the compressor efficiency, the compressed pulse energy can be as high as $95 \mathrm{~mJ}$.

Figure 8 illustrates the temporal contrast of the amplified laser pulse between $-500 \mathrm{ps}$ and $+150 \mathrm{ps}$ by using a third-order femtosecond cross-correlator (Sequoia, Amplitude Systems) at maximum pulse energy of multi-pass amplifier. Data points were recorded with 1 ps time steps and 10 shots averaging. The detection limit was $7.2 \times 10^{-9}$ in this study. The contrast level at -150 ps before the sub-picosecond (main) laser pulse was measured to be $7.2 \times 10^{-9}$, and therefore we consider that the temporal contrast of this laser pulse can be less than $7.2 \times 10^{-9}$. We attribute the extremely high temporal contrast on the picosecond time scale with the minimization of the parametric fluorescence in the OPCPA preamplifier.

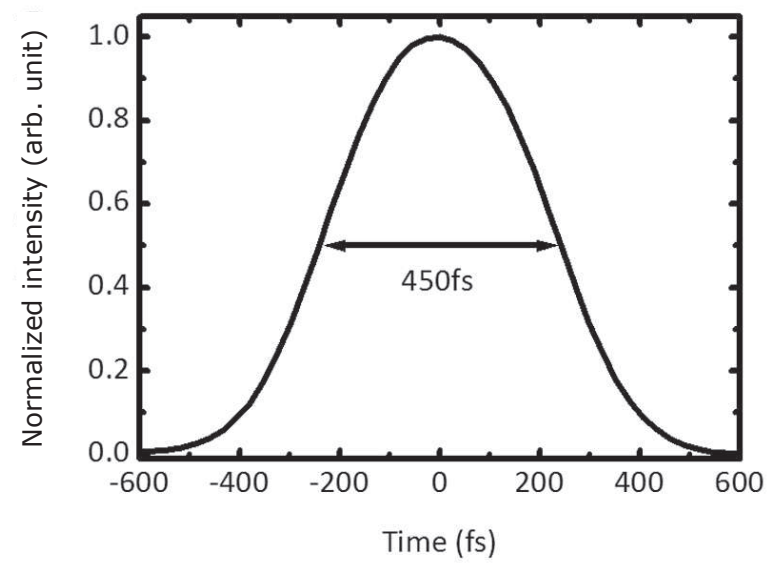

Fig. 7 The autocorrelation trace of an amplified laser pulse measured by a commercial FROG. The pulse duration is $450 \mathrm{fs}$.

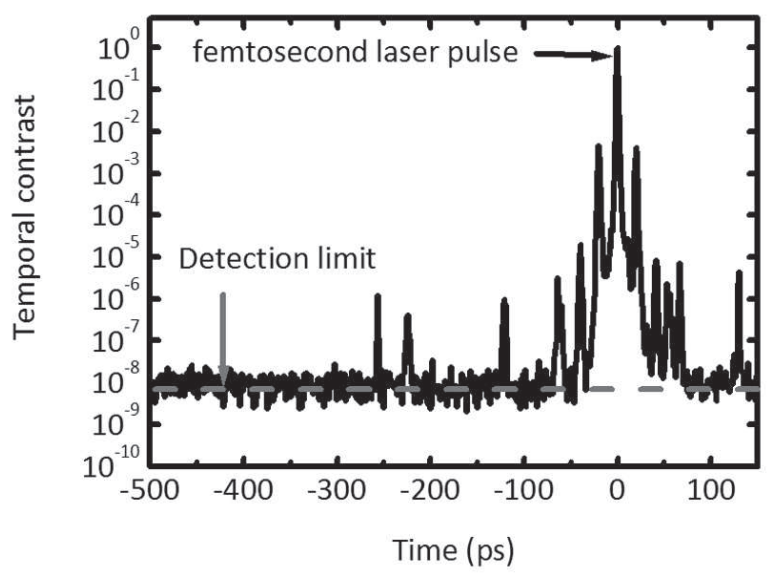

Fig. 8 Temporal contrast of the recompressed laser pulse is measured by a third-order autocorrelator. The black solid curve shows the temporal contrast of femtosecond laser pulse. The gray dash line shows the detection limit of this measurement. The detection limit is measured to be $7.2 \times 10^{-9}$. 
Also shown in Fig. 8, there are several peaks observed at $-37 \mathrm{ps},-19 \mathrm{ps}, 19 \mathrm{ps}$, and $37 \mathrm{ps}$. These signals before and after amplified main pulse are attributed to front and back surface reflections from the Yb:YAG thin disk surface (refractive index of YAG: 1.82, incidence angle: $\sim 2$ degree). Unless these unwanted signals reflect back to thin disk in next 1-pass amplification, they would be removed. To shift the laser beam by $4.5 \mathrm{~mm}$ on the disk surface after 1 pass, the wedge angle is set to 0.09 degree. Therefore, we remove the surface reflection prepulse with a 0.1 degree wedge disk in our multipass amplifier configuration.

\section{Conclusion}

In conclusion, we report the OPCPA/ Yb:YAG ceramic thin disk laser system having the hundred $\mathrm{mJ}$ level with sub-picosecond pulse duration and high contrast level at $10 \mathrm{~Hz}$ repetition-rate. The output energy of $130 \mathrm{~mJ}$ with the spectral bandwidth of $2.5 \mathrm{~nm}$ was obtained from the multi-pass amplifier was achieved at a LD pump energy of $1.4 \mathrm{~J}$. The final recompressed laser pulse duration was measured to be $450 \mathrm{fs}$. The contrast level of this laser pulse was measured to be less than $7.2 \times 10^{-9}$ at $-150 \mathrm{ps}$. This laser is one of the most promising candidates for high field laser-matter experiments for investigating the laser peening mechanism.

\section{Acknowledgement}

This work was partly supported by the "Consortium for Photon Science and Technology (C-PhoST)" program funded by the Special Coordination Funds for Promoting Science and Technology commissioned by Ministry of Education, Culture, Sports, Science and Technology (MEXT) of Japan. It was also partly the Special Coordination Fund (SCF) for Promoting Science and Technology commissioned by the MEXT of Japan. The authors thank T. Shimomura, M. Tanoue, Y. Nakai for technical supports, and A. Yogo, M. Tampo, T. Hori, H. Sakaki, K. Nagashima, H. Daido, and T. Tajima for helpful discussions.

\section{References}

1) P. Peyre, R. Fabbro, P. Merrien, and H. P. Lieurade: Mater. Sci Eng. A 210 (1996) 102.

2) Y. Sano, N. Mukai, K. Okazaki, M. Obata: Nucl. Instrum. Meth. Phys. Res. B 121 (1997) 432.

3) H. Nakano, S. Miyauti, N. Butani, T. Shibayanagi, M. Tsukamoto, and N. Abe: J. Laser Micro/Nanoeng. 4 (2009) 35.

4) A. Yogo, H. Daido, S. V. Bulanov, K. Nemoto, Y. Oishi, T. Nayuki, T. Fujii, K. Ogura, S. Orimo, A. Sagisaka, et al.: Phys. Rev. E 77 (2008) 016401.

5) H. Kiriyama, M. Mori, Y. Nakai, T. Shimomura, M. Tanoue, A. Akutsu, S. Kondo, S. Kanazawa, H. Okada, T. Motomura, et al.: Opt. Lett. 33 (2008) 645.

6) H. Yoshida, E. Ishii, R. Kodama, H. Fujita, Y. Kitagawa, Y. Izawa, and T. Yamanaka: Opt. Lett. 28 (2003) 257.

7) H. Kiriyama, M. Mori, Y. Nakai, T. Shimomura, H. Sasao, M. Tanoue, S. Kanazawa, D. Wakai, F. Sasao, H. Okada, et al.: Opt. Lett. 35 (2010) 1497.

8) I. Jovanovic, Ch. A. Ebbers, and C. P. J. Barty: Opt. Lett. 27 (2002) 1622.

9) I. O. Musgrave, C. Hernandez-Gomez, D. Canny, J. Collier, and R. Heathcote: Appl. Opt. 46 (2007) 6978

10) C. Dorrer, I. A. Begishev, A. V. Okishev, and J. D. Zuegel: Opt. Lett. 32 (2007) 2143.

11) E. W. Gaul, M. Martinez, J. Blakeney, A. Jochmann, M. Ringuette, D. Hammond, T. Borger, R. Escamilla, S. Douglas, W. Henderson, et al:: Appl. Opt. 49 (2010) 1676.

12) A. Yogo, H. Kiriyama, M. Mori, T. Zh. Esirkepov, K. Ogura, A. Sagisaka, S. Orimo, M. Nishiuchi, A. S. Pirozhkov, H. Nagatomo, et al.: Eur. Phys. J. D 55 (2009) 421.

13) T. Y. Fan, IEEE J Quant. Electron. 28 (1992) 2692.

14) K. Takaichi, H. Yagi, J. Lu, A. Shirakawa, K. Ueda, T. Yanagitani, A. A. Kaminskii: Phys. Stat. Sol. A 200 (2003) R5.

15) M. Tsunekane and T. Taira: Appl. Phys. Lett. 90 (2007) 21101.

16) J. Hein, M. C. Kaluza, R. Bödefeld, M. Siebold, S. Podleska and R. Sauerbrey: Lect. Notes Phys. 694 (2006) 47.

17) M. Siebold, M. Hornung, R. Boedefeld, S. Podleska, S. Klingebiel, Ch. Wandt, F. Krausz, S. Karsch, R. Uecker, A. Jochmann, et al.: Opt. Lett. 33 (2008) 2770.

18) J. Tümmler, R. Jung, H. Stiel, P. V. Nickles, and W. Sandner: Opt. Lett. 34 (2009) 1378.

19) T. Metzger, A. Schwarz, C. Y. Teisset, D. Sutter, A. Killi, R. Kienberger, F. Krausz: Opt. Lett. 34 (2009) 2123.

20) M. Suzuki, H. Kiriyama, I. Daito, H. Okada, Y. Nakai, S. Orimo, M. Sato, Y. Tamaoki, T. Yoshii, J. Maeda, et al.: Appl. Phys. B 97 (2009) 379 .

21) A. Giesen and J. Speiser: IEEE JSTQE 13 (2007) 598. 